\title{
Correlation between the high-temperature local mobility of heterocyclic polyimides and their mechanical properties
}

Victor M. Nazarychev, ${ }^{1}$ Alexey V. Lyulin, ${ }^{2}$ Sergey V. Larin, ${ }^{1}$ Iosif V. Gofman, ${ }^{1}$ José M.

Kenny, ${ }^{1,3}$ Sergey V. Lyulin ${ }^{* 1}$

${ }^{1}$ Institute of Macromolecular Compounds, Russian Academy of Sciences, Bol'shoi pr. 31 (V.O.), 199004

St. Petersburg, Russia

${ }^{2}$ Theory of Polymers and Soft Matter Group, Technische Universiteit Eindhoven, PO Box 513, 5600 MB Eindhoven, The Netherlands

${ }^{3}$ Materials Science and Technology Centre, University of Perugia, Loc. Pentima, 4, 05100 Terni, Italy

Correspondence to: s.v.lyulin@gmail.com 


\section{Local orientational mobility of different phenylene rings in PI R-BAPS samples with and without partial charges}

We studied the local orientational mobility not only of the fastest last ring of the $8^{\text {th }}$ repeating unit in each chain of the R-BAPS polyimide (PI) sample with and without partial charges but also of all the other phenylene rings in all R-BAPS molecules, Fig. S1.

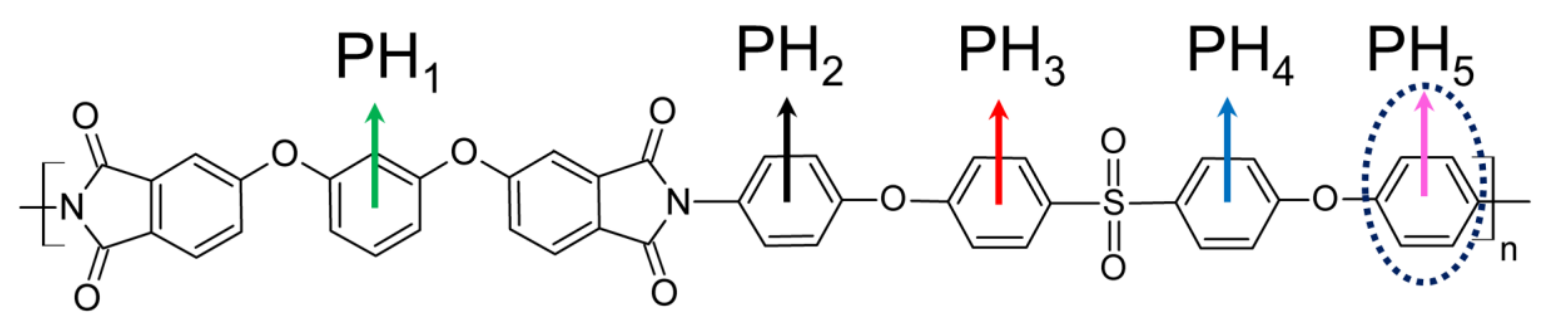

Fig. S1. Repeating unit of the PI R-BAPS. Unit vectors normal to the phenylene rings $\mathrm{PH}_{1}-\mathrm{PH}_{5}$ investigated in the present simulation are shown by arrows. The last ring is shown inside the dotted line (considered for the $8^{\text {th }}$ repeating unit).

The analysis of the results leads to the following conclusions. First of all, the mobility of the last rings is the highest, Fig. S2. 

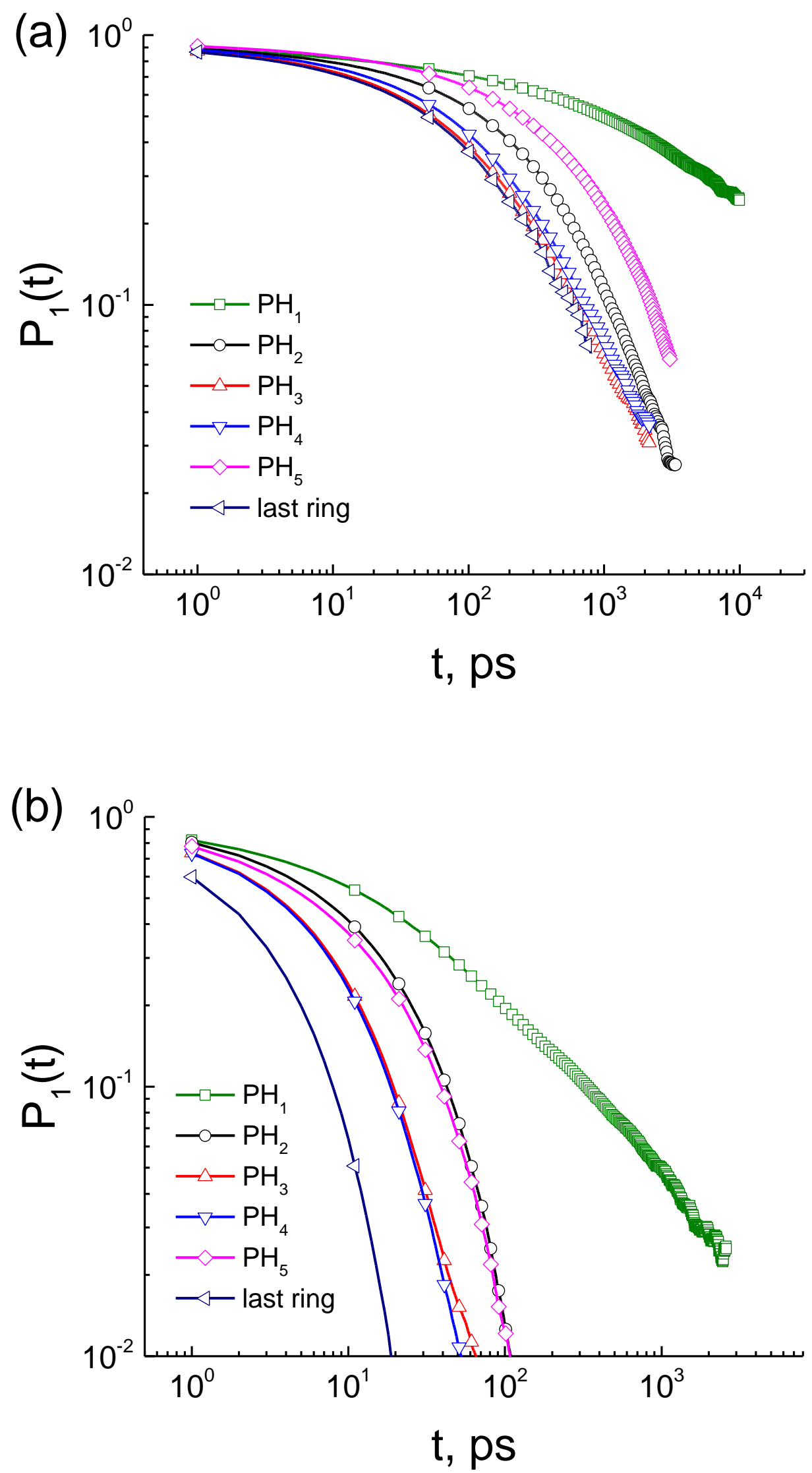

Fig. S2. Time dependence of the autocorrelation functions $P_{1}(t)$ for unit vectors shown in Fig. S1 in PI RBAPS samples with (a) and without (b) partial charges at $\mathrm{T}=800 \mathrm{~K}$. 
Time dependence of $P_{1}(t)$ functions have been calculated at different temperatures above glass transition for all the considered phenylene rings in PI R-BAPS samples with and without partial charges, see Figs. S3 and S4 correspondingly.

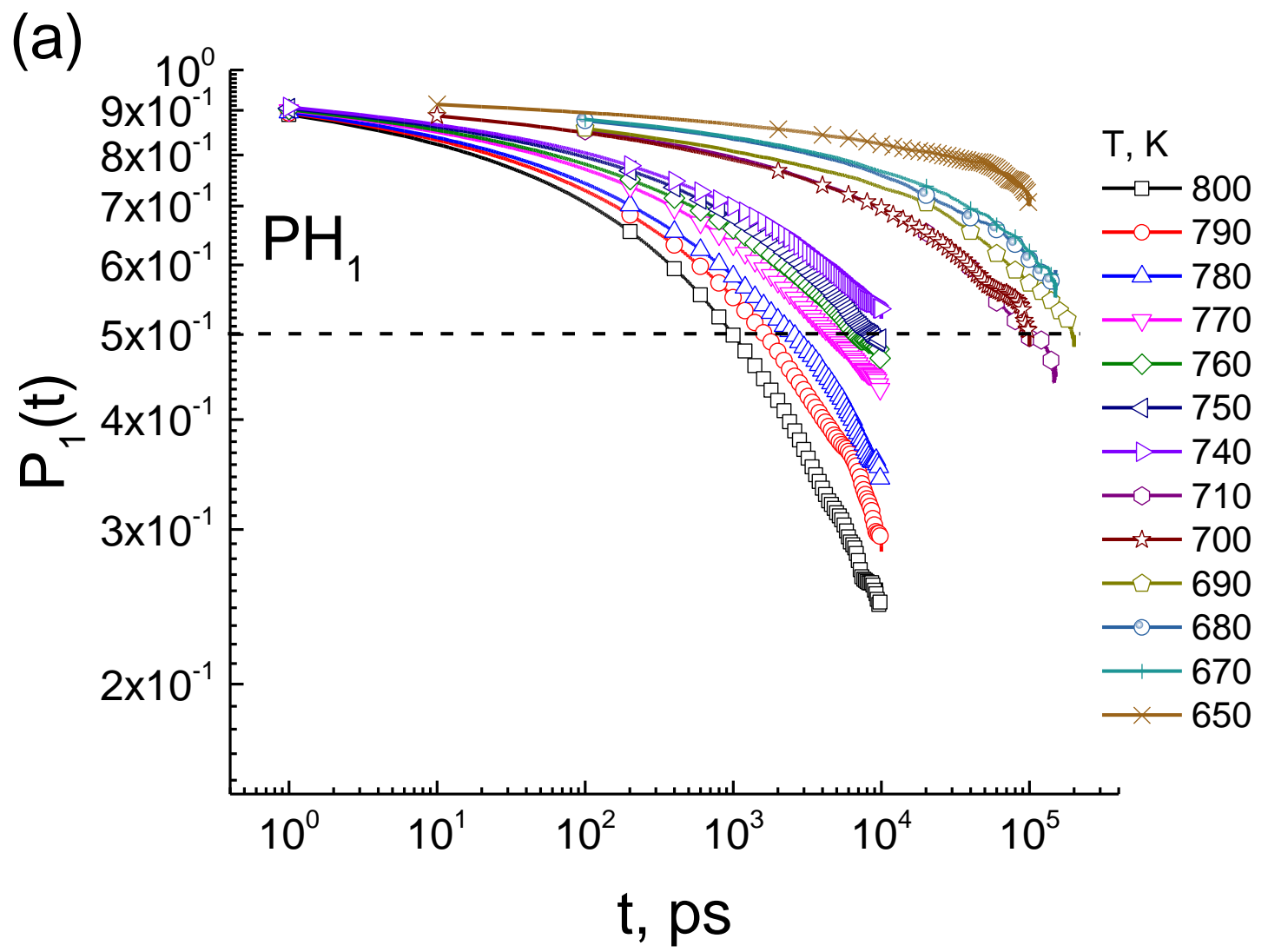



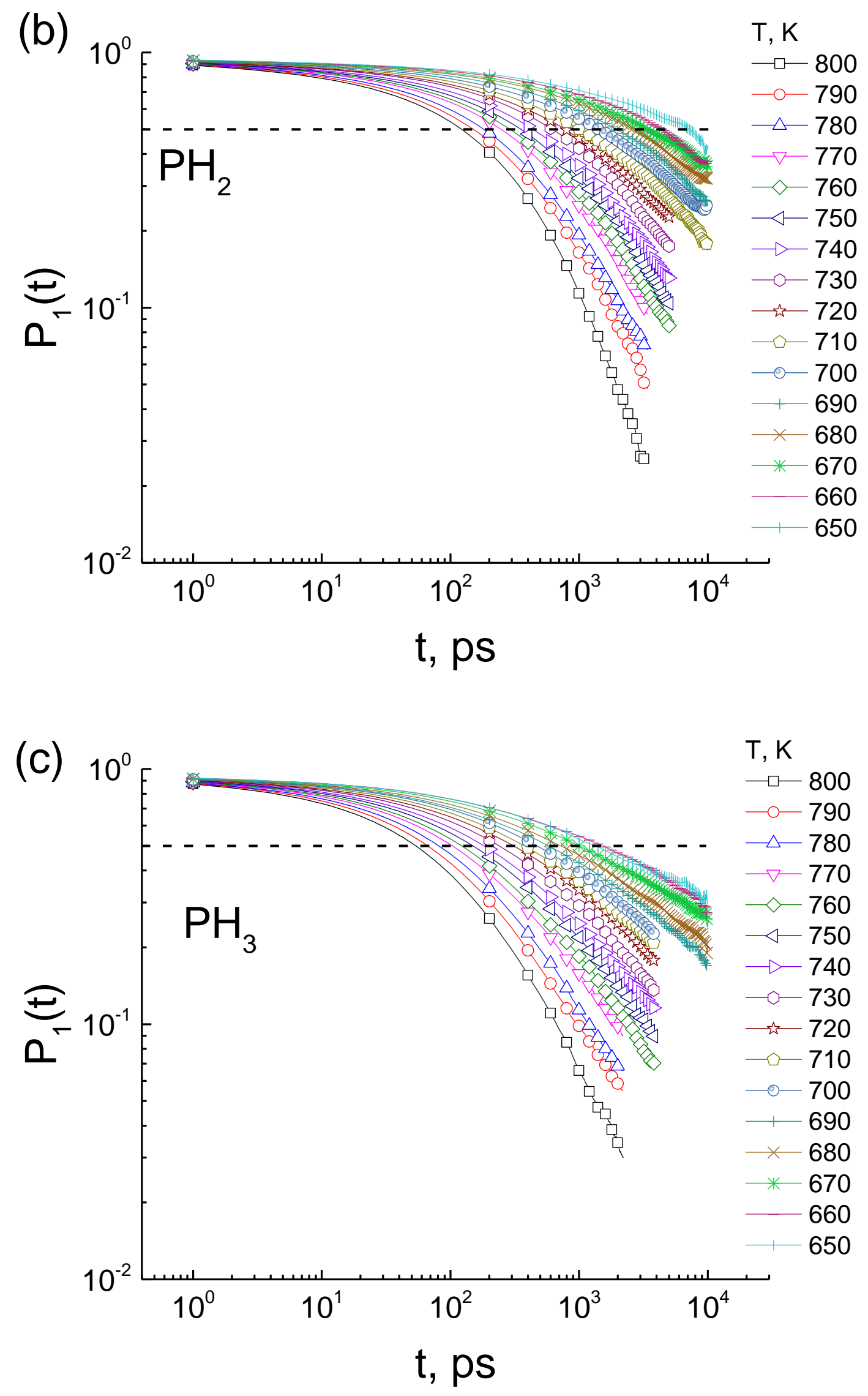

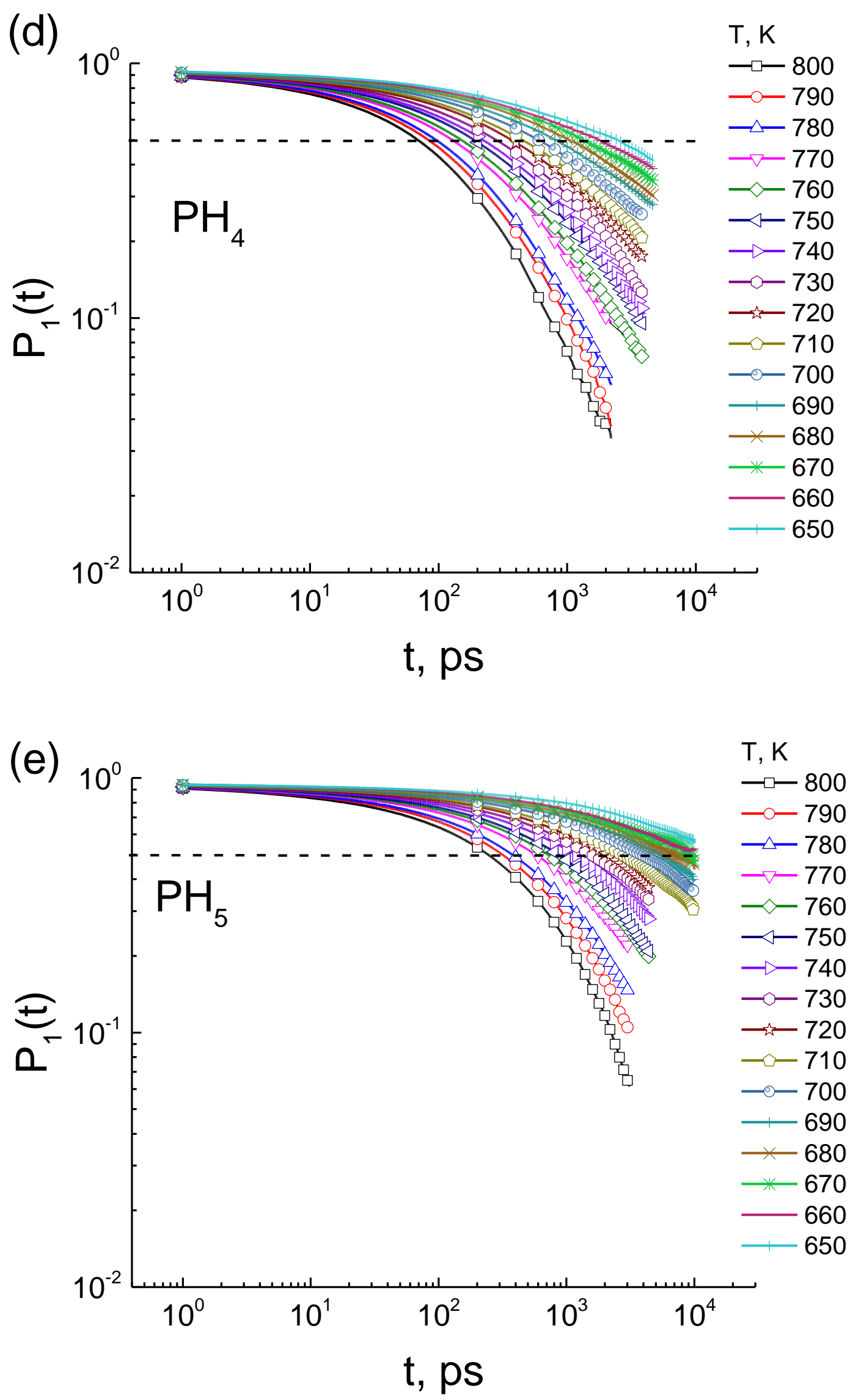


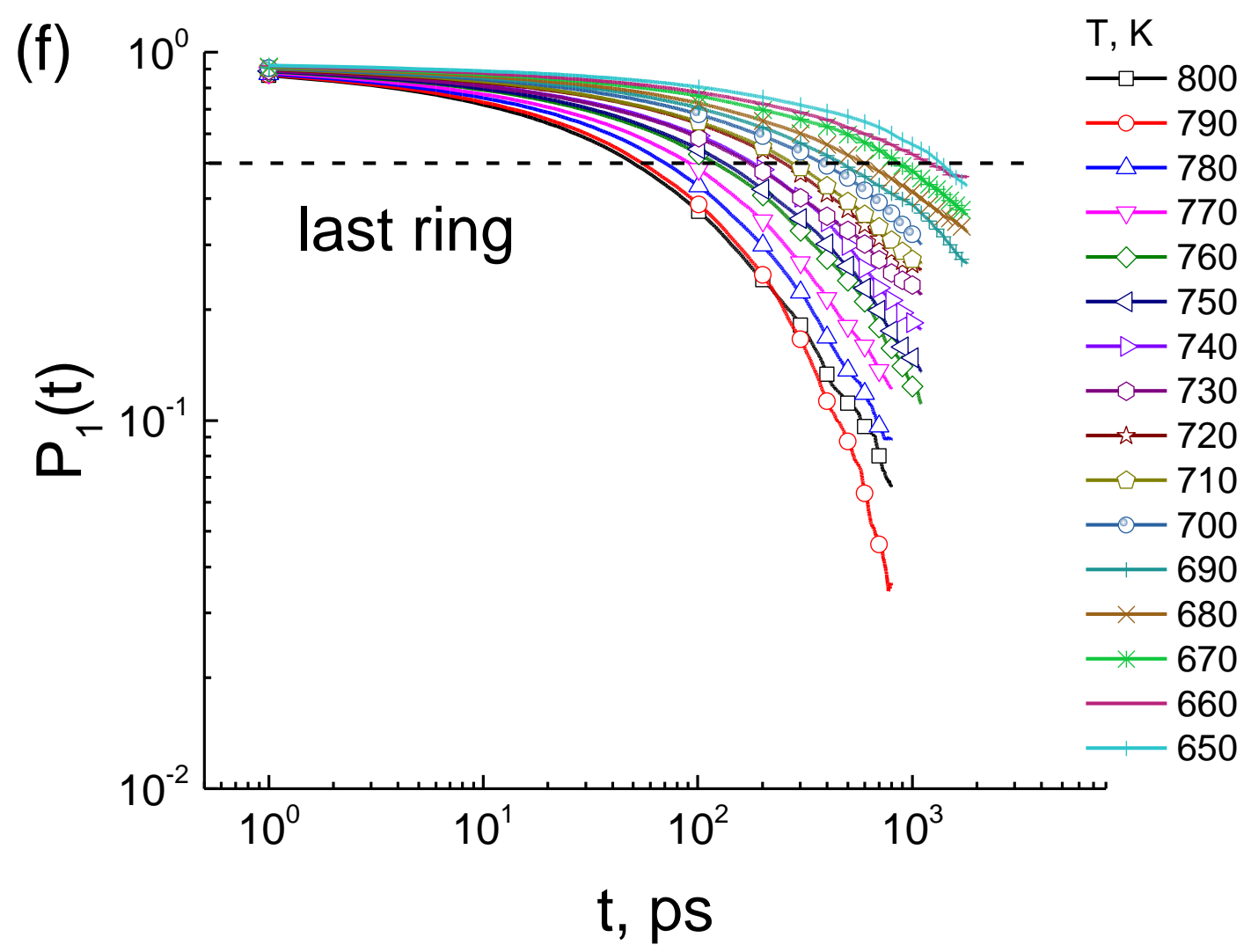

Fig. S3. Time dependence of $P_{1}(t)$ functions at different temperatures above glass transition for all the considered phenylene rings in PI R-BAPS samples with partial charges: (a) $\mathrm{PH}_{1}$, (b) $\mathrm{PH}_{2}$, (c) $\mathrm{PH}_{3}$, (d) $\mathrm{PH}_{4}$, (e) $\mathrm{PH}_{5}$, and (f) last ring of the $8^{\text {th }}$ repeating unit. The value $P_{1}(t)=0.5$ is represented by the dashed line. 

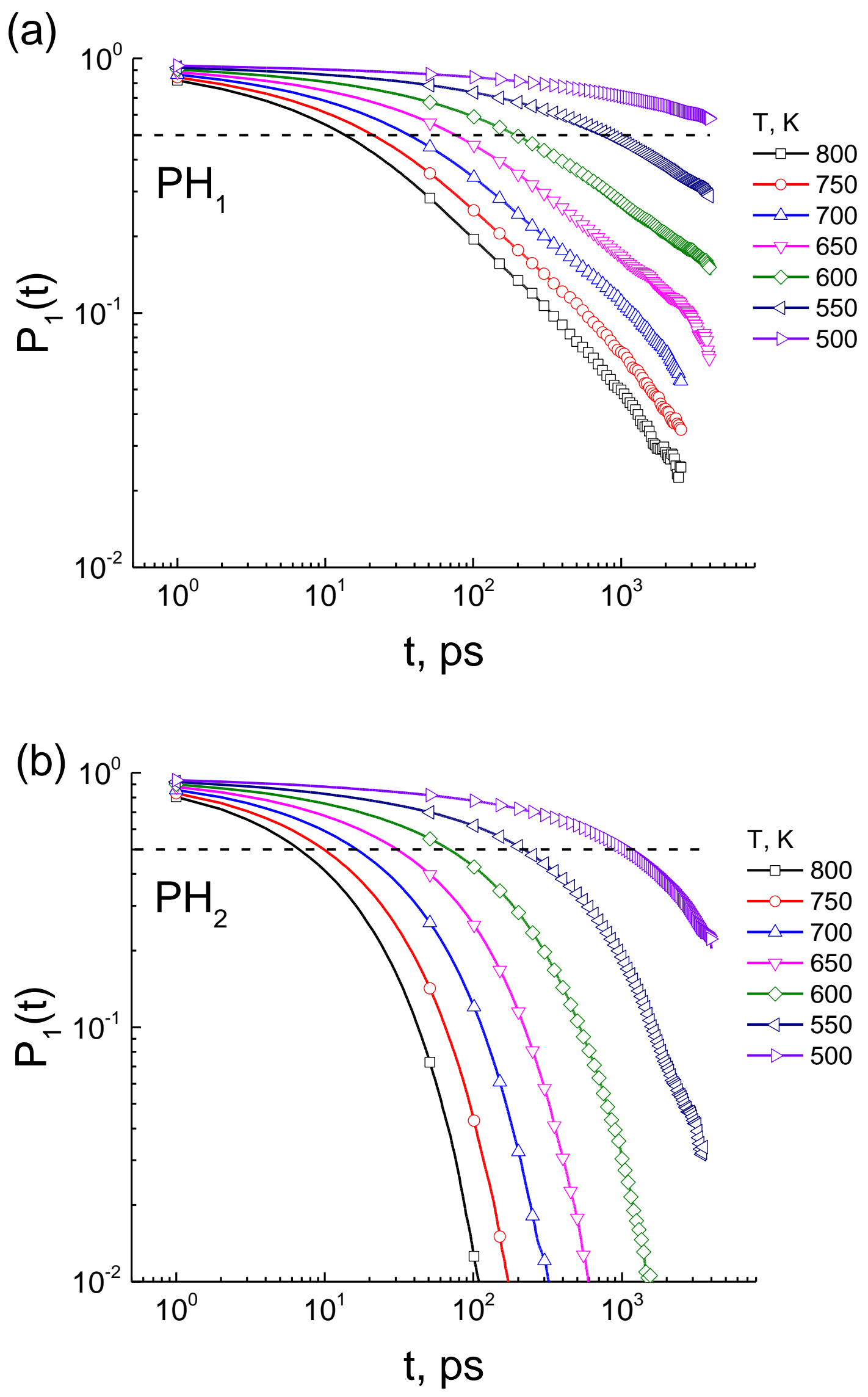

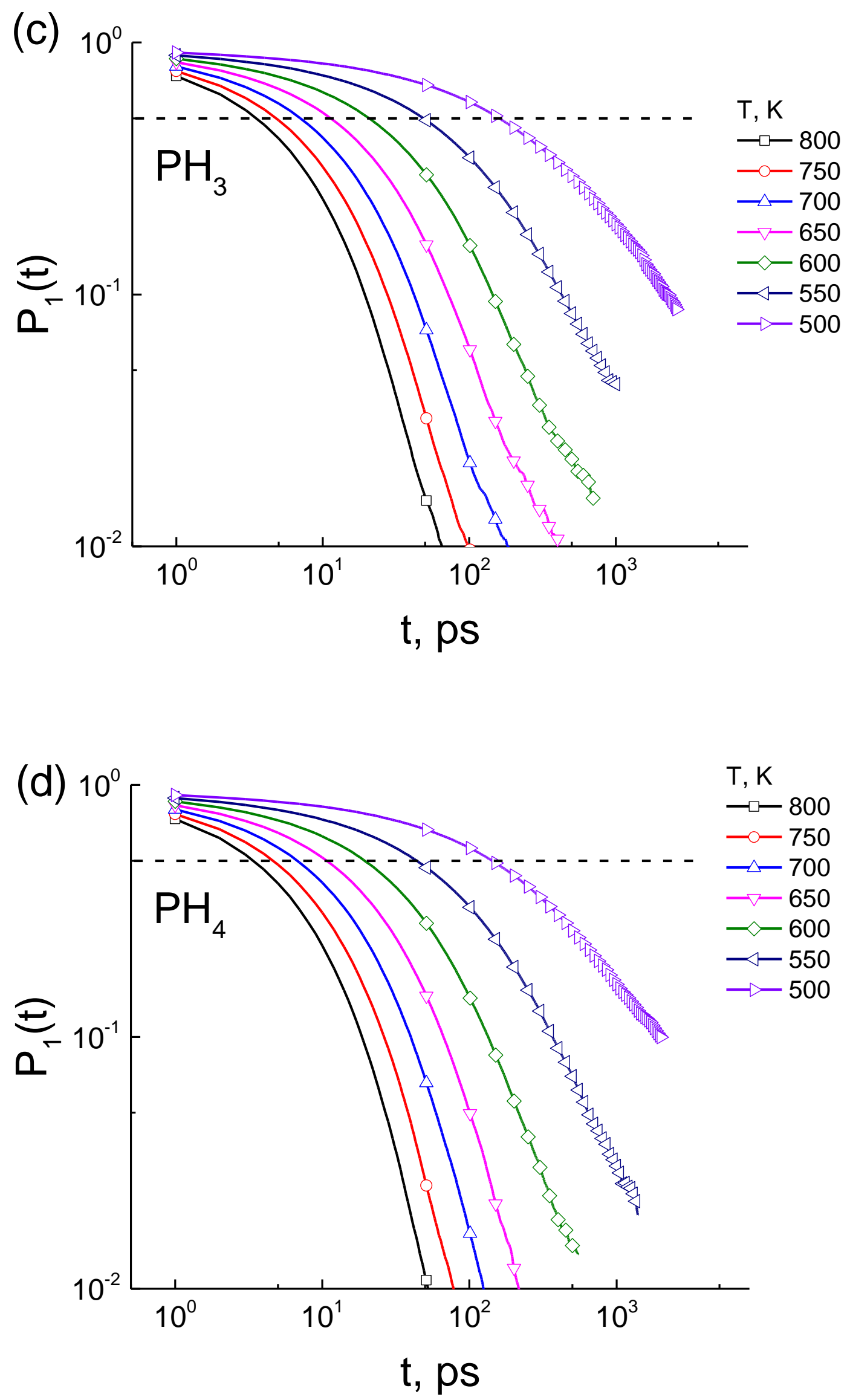

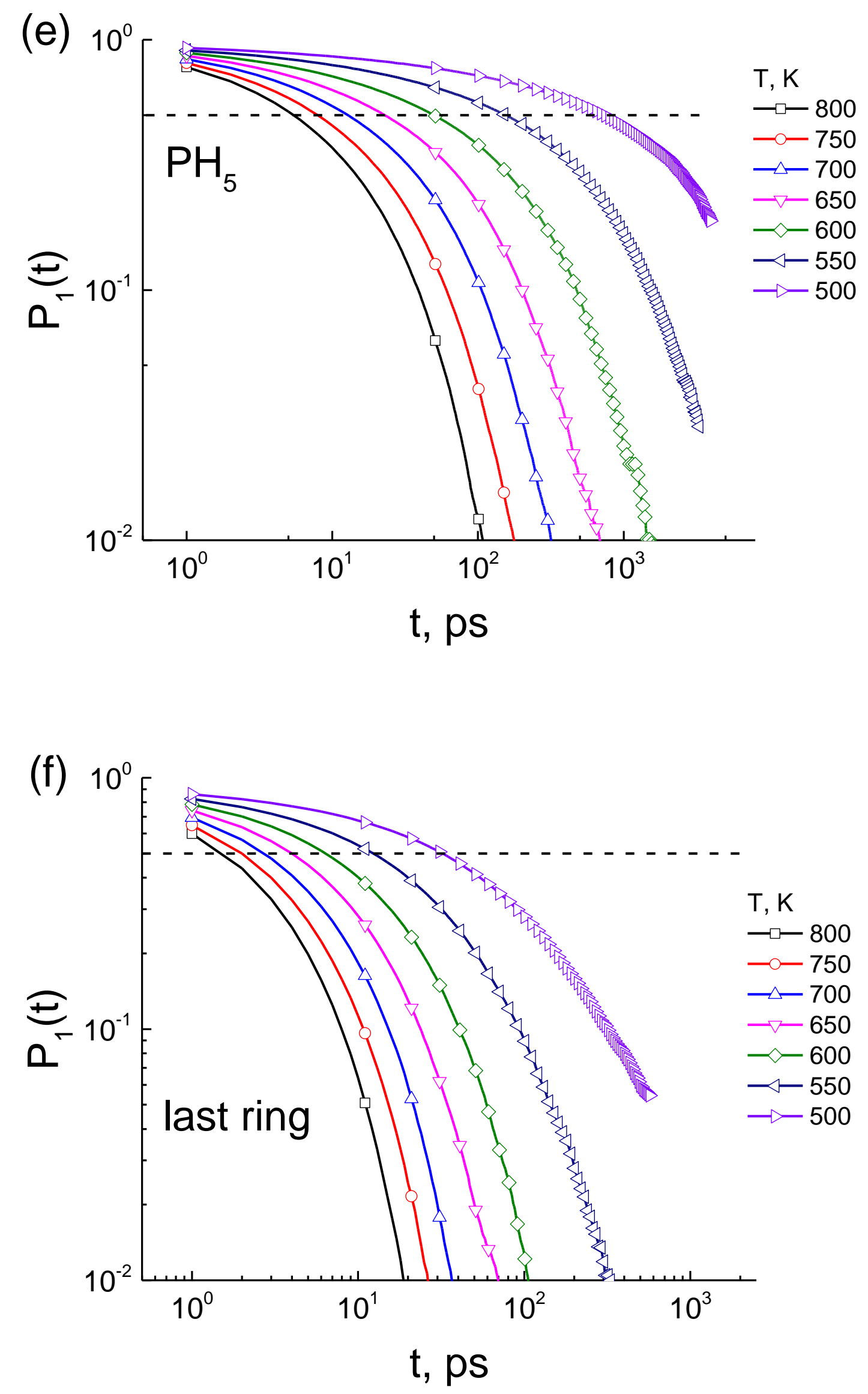
Fig. S4. Time dependence of $P_{1}(t)$ functions at different temperatures above glass transition for all the considered phenylene rings in PI R-BAPS samples without partial charges: (a) $\mathrm{PH}_{1}$, (b) $\mathrm{PH}_{2}$, (c) $\mathrm{PH}_{3}$, (d) $\mathrm{PH}_{4}$, (e) $\mathrm{PH}_{5}$, and (f) last ring of the $8^{\text {th }}$ repeating unit. The value $P_{1}(t)=0.5$ is represented by the dashed line.

The time dependences $P_{1}(t)$ were approximated using the KWW stretched exponential functions $P_{1}(t)=A \exp (-(t / \tau))^{\beta}$, where $\mathrm{A} \leq 1, \tau$ is the characteristic relaxation time, $\beta$ is a parameter taking into account the non-exponentiality of the relaxation process. 

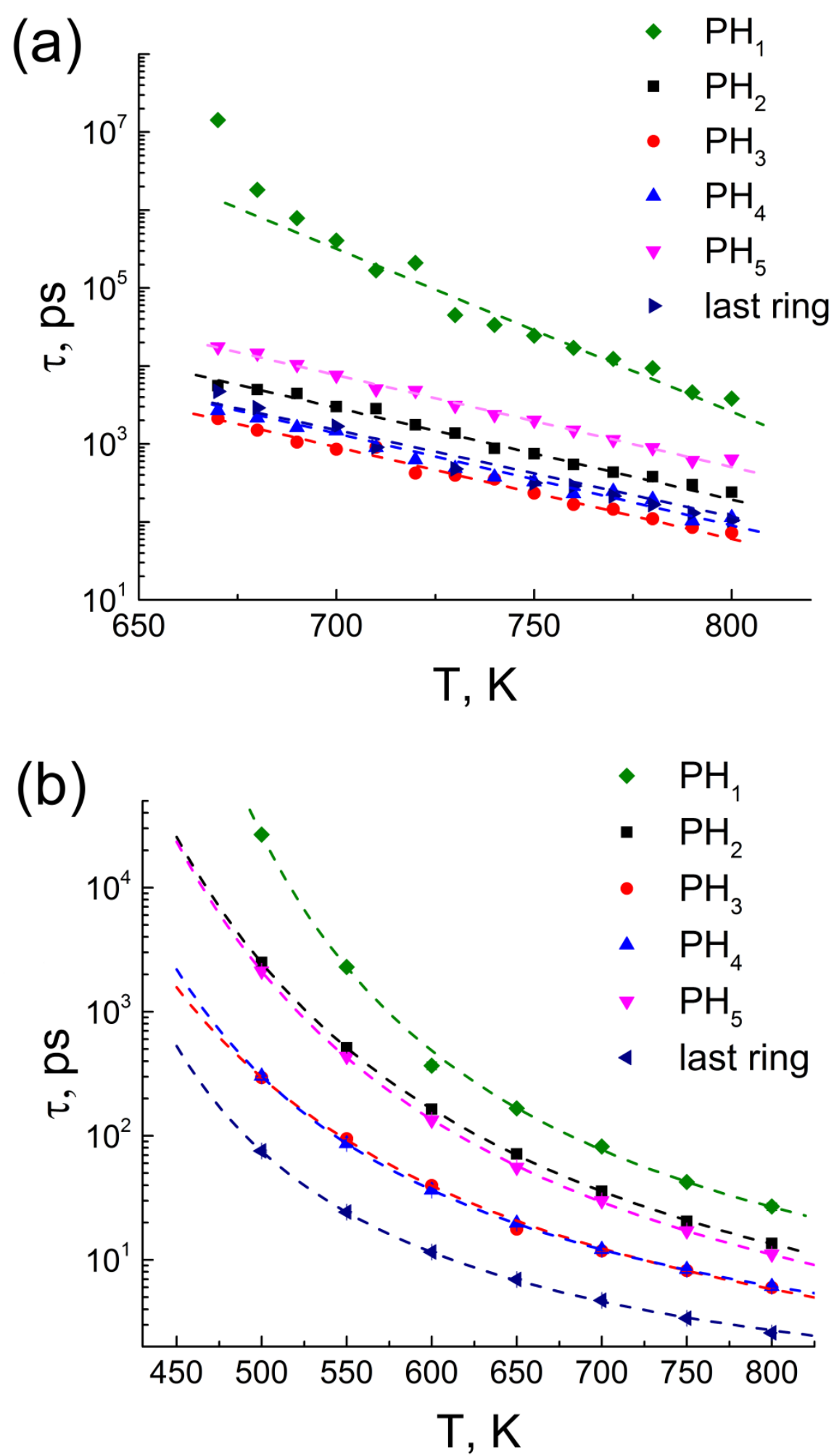

Fig. S5. Temperature dependence of the relaxation time $\tau$ for $P_{1}(t)$ autocorrelation functions for different phenylene rings $\left(\mathrm{PH}_{1}-\mathrm{PH}_{5}\right)$ for the samples with (a) and without (b) partial charges. The dashed lines on figures $\mathrm{S} 5(\mathrm{a})$ and $\mathrm{S} 5(\mathrm{~b})$ represent the Arrhenius $\tau \approx \tau_{0} \exp \left[E_{a} / R T\right]$ and Vogel-Fulcher-Tammann (VFT) $\tau \approx \tau_{0} \exp \left[E_{a} / R\left(T-T_{0}\right)\right]$ approximations respectively. 
The obtained results have shown that symmetrically located rings as $\mathrm{PH}_{3}$ and $\mathrm{PH}_{4}$ or $\mathrm{PH}_{2}$ and $\mathrm{PH}_{5}$ have almost identical relaxation times. It should be noted that local orientation mobility of the $\mathrm{PH}_{1}$ phenylene ring located in a dianhydride fragment of the PI R-BAPS repeating unit is the slowest in comparison with all the other considered rings. This can be caused by the fact that, most likely, the $\mathrm{PH}_{1}$ phenylene ring located in a polymeric chain in meta-position that, most likely, considerably limits the angle of its rotation around adjacent oxygen atoms and leads to slowing down its mobility.

Rather strong heterogeneity of the dynamics has been observed in the relaxation of the phenyl rings in both samples. The temperature dependence of the relaxation times can be fit by the VFT dependence for samples without partial charges. The activation energy $E_{a}^{\prime}$ and the values of the critical temperature $T_{0}$ are different for different phenylene rings, this could reflect the cooperative nature of the vitrification. For the samples without partial charges the critical temperatures are very low, about 120-200 K below the corresponding glass transition, see. Table S1.

The time dependence of the autocorrelation function $P_{1}(t)$ of the unit vector normal to the surface of the selected phenylene ring was fitted by VFT (Vogel-Fulcher-Tammann) dependence for the samples without partial charges. Obtained values of $\tau_{0}$, activation energy $E_{a}$, and critical temperature $T_{0}$ for the PI R-BAPS sample without partial charges are reported in Table S1.

Table S1. VFT function parameters used to approximate the temperature dependence of the relaxation times for the PI R-BAPS sample without partial charges.

\begin{tabular}{|c|c|c|c|}
\hline Phenylene's ring & $\tau_{0}, \mathrm{ps}$ & $E_{a}^{\prime}, \mathrm{kJ} / \mathrm{mol}$ & $T_{0}, \mathrm{~K}$ \\
\hline $\mathrm{PH}_{1}$ & $0.53 \pm 0.13$ & $15 \pm 1$ & $330 \pm 5$ \\
\hline $\mathrm{PH}_{2}$ & $0.15 \pm 0.02$ & $21 \pm 1$ & $242 \pm 4$ \\
\hline $\mathrm{PH}_{3}$ & $0.32 \pm 0.05$ & $14 \pm 2$ & $272 \pm 2$ \\
\hline $\mathrm{PH}_{4}$ & $0.35 \pm 0.01$ & $13 \pm 1$ & $278 \pm 2$ \\
\hline $\mathrm{PH}_{5}$ & $0.14 \pm 0.02$ & $20 \pm 1$ & $249 \pm 6$ \\
\hline last ring & $0.33 \pm 0.02$ & $9 \pm 1$ & $311 \pm 3$ \\
\hline
\end{tabular}

For the samples with partial charges the much smaller deviations from the Arrhenius temperature dependence of the $\mathrm{PH}_{2}-\mathrm{PH}_{5}$ rings relaxation could reflect the important result of the strong dipoledipole interactions between the sulfone groups. 


\section{Local orientational mobility in PI R-BAPS samples with and without partial charges at similar density values}

The study compared the local mobility in the PI R-BAPS samples with and without partial charges. Simulations with partial charges resulted in noticeably denser system and therefore the observed slowing down of dynamics could be only a manifestation of a higher density of the sample. To check this suggestion we increased the density of the samples of PI R-BAPS without partial charges to match the density of the PI R-BAPS sample with partial charges. The periodic cell of the sample without partial charges has been isotropically compressed at temperature above $T_{g}$ with the rate of $\gamma_{\mathrm{d}}=1.8 \times 10^{8} \mathrm{~s}^{-1}$ in the NPT ensemble, using the intrinsic deform function of Gromacs. The compression stopped when the density became equal to that for the sample with partial charges at the same temperature. The pressure was controlled using the Berendsen barostat. After the necessary equilibration, the NVT MD run has been carried out for $4 \mathrm{~ns}$, and the $P_{1}(t)$ autocorrelation function has been analyzed, Fig. S6.

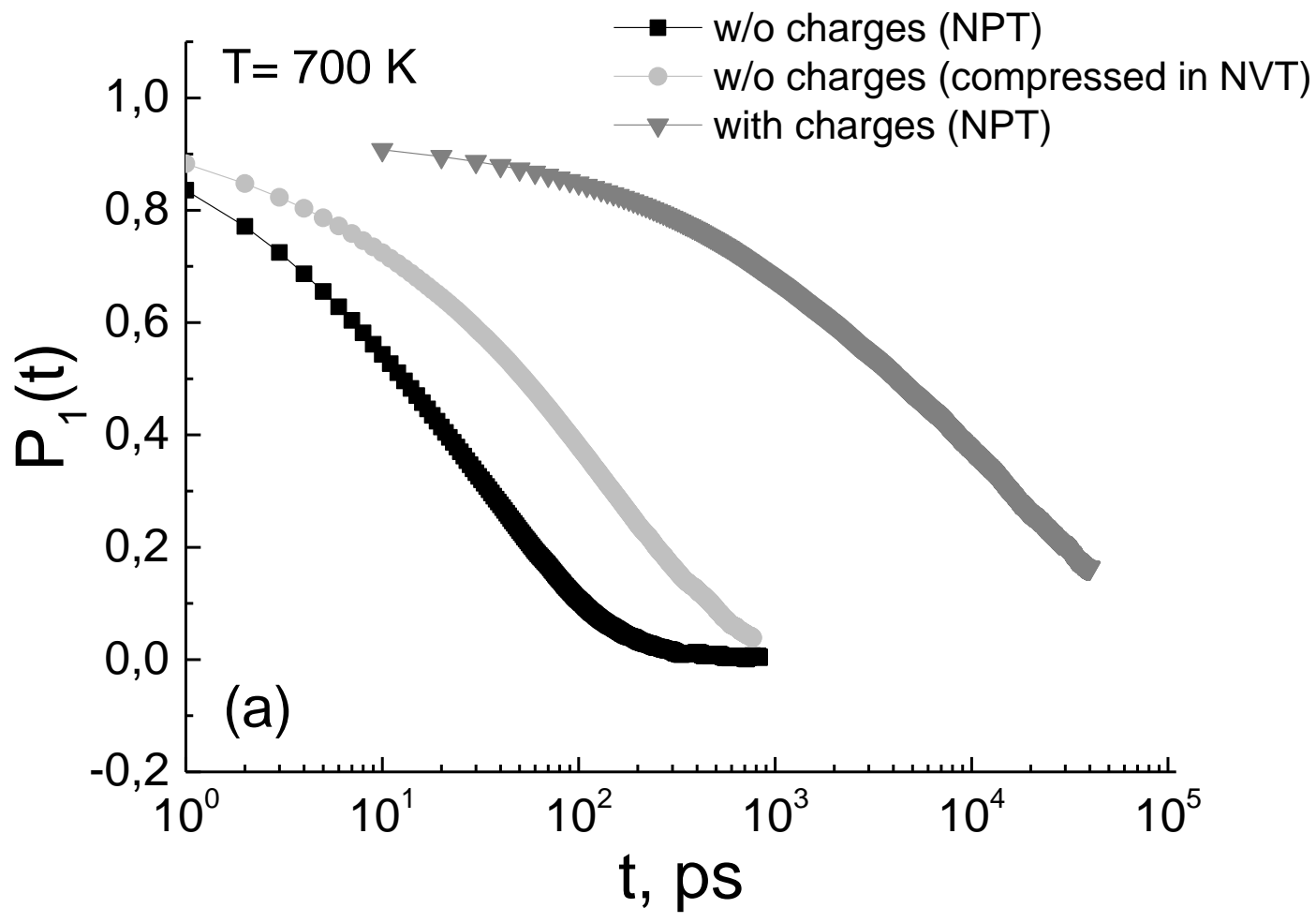




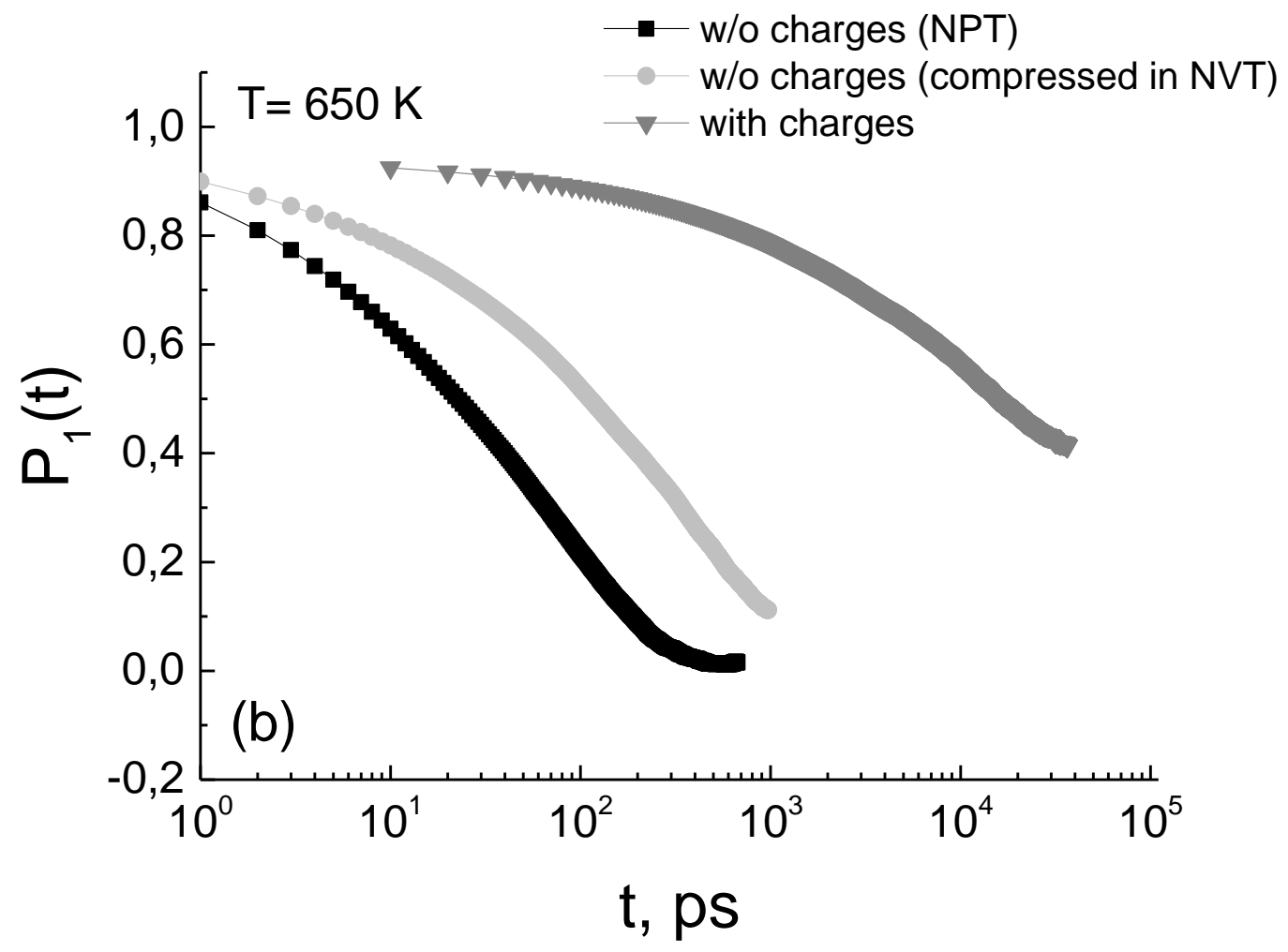

Fig. S6. Time dependence of the autocorrelation function $P_{1}(t)$ of the unit vector normal to the surface of the phenylene ring $\mathrm{PH}_{5}$ for the samples without partial charges (black squares), with artificially increased density by deformation in NVT ansemble (light gray circles) and with partial charges (gray invert triangles) at (a) $700 \mathrm{~K}$ and (b) $650 \mathrm{~K}$.

The results are presented in SI, Fig S6. The local segmental mobility of PI R-BAPS fragments without partial charges is (not surprisingly) slowing down upon the density increase. At the same time this local segmental mobility still remains much larger (at the same temperature) as compared to that for the sample with partial charges. We conclude that the local orientational mobility of the samples with partial charges is much smaller as compared to those without partial charges, not only because of the difference in density, but also because of the significant influence of the dipole-dipole interactions. These interactions - most probably - lead to the significant heterogeneity of the free-volume distribution. 